University of Nebraska - Lincoln

DigitalCommons@University of Nebraska - Lincoln

P. F. (Paul Frazer) Williams Publications

Electrical \& Computer Engineering, Department

April 1994

\title{
Interferometric determination of the quadratic electro-optic coefficient of nitrobenzene
}

\author{
Harshad P. Sardesai \\ University of Texas at Arlington \\ William C. Nunnally \\ University of Texas at Arlington \\ P. F. Williams \\ University of Nebraska - Lincoln, pfw@moi.unl.edu
}

Follow this and additional works at: https://digitalcommons.unl.edu/elecengwilliams

Part of the Electrical and Computer Engineering Commons

Sardesai, Harshad P.; Nunnally, William C.; and Williams, P. F., "Interferometric determination of the quadratic electro-optic coefficient of nitrobenzene" (1994). P. F. (Paul Frazer) Williams Publications. 1. https://digitalcommons.unl.edu/elecengwilliams/1

This Article is brought to you for free and open access by the Electrical \& Computer Engineering, Department of at DigitalCommons@University of Nebraska - Lincoln. It has been accepted for inclusion in P. F. (Paul Frazer) Williams Publications by an authorized administrator of DigitalCommons@University of Nebraska - Lincoln. 


\title{
Interferometric determination of the quadratic electro-optic coefficient of nitrobenzene
}

\author{
Harshad P. Sardesai, William C. Nunnally, and P. F. Williams
}

\begin{abstract}
An interferometric method that was used to measure the electro-optic $s_{11}$ coefficient of nitrobenzene is presented. The method uses a Mach-Zehnder interferometer arrangement that produces a finite fringe interferogram. A nitrobenzene Kerr cell is placed in one arm of the interferometer and a pulsed high voltage is applied to the two rectangular electrodes. The voltage application causes the fringe image to shift in time, and this fringe shift is measured by a streak camera that streaks the fringe image across a slit. The streak start time is chosen before the application of the voltage pulse and ends after the voltage pulse has been applied. Thus continuous observation of the event is possible. By accurately measuring the fringe shifts the electro-optic $s_{11}$ coefficient can be determined. The experimental arrangement is described, and the results obtained are discussed.
\end{abstract}

\section{Introduction}

Nitrobenzene has been extensively used as the electrooptic medium in Kerr cells. ${ }^{1-6}$ Such Kerr cells were used primarily for the measurement of high-voltage pulses and high electric fields ${ }^{3,4}$ and as optical shutters. ${ }^{5,6}$ Most of the experiments with nitrobenzene that were reported previously used the approach in which the incident linearly polarized light beam was passed through the Kerr cell with the plane of polarization at $45^{\circ}$. In these experiments an external electric field was applied to the electrodes of the Kerr cell, and the phase retardation between the parallel and perpendicular components of the light electric-field vector (resulting from the induced electrooptic effect) was measured either by using a crossed polarizer arrangement ${ }^{3}$ or by interferometric techniques. ${ }^{4}$ This phase retardation was then related to the applied electric field with the well-documented Kerr constant, and the unknown parameters were determined. To our knowledge, none of the experiments reported so far has used the absolute retardation of only one component of the electric-field vector. Such a measurement would require knowledge of the

H. P. Sardesai and W. C. Nunnally are with the Applied Physical Electronics Research Center, University of Texas at Arlington, Arlington, Texas 76019-0308. P. F. Williams is with the Department of Electrical Engineering, University of Nebraska-Lincoln, Lincoln, Nebraska 68588-0511.

Received 3 August 1992, revised manuscript received 5 August 1993.

0003-6935/94/101791-04\$06.00/0.

(c) 1994 Optical Society of America. electro-optic coefficients of nitrobenzene. Our experiment was motivated during the development of a semiconductor surface-field measurement technique that used the Kerr electro-optic effect and the MachZehnder interferometric analyzer. As we had reported earlier, ${ }^{7}$ we first used the conventional approach of passing both of the polarization states through the Kerr cell and measuring the phase retardation between the two states, which would then help us determine the electric field. This approach was not entirely successful in determining the surface fields ${ }^{8}$ and this led us to use the approach of passing only one polarization state through the Kerr cell and use the other polarization state as a reference. ${ }^{9}$ This approach required knowledge of the $s_{11}$ electro-optic coefficient of nitrobenzene, which was measured as part of the Kerr cell calibration process.

\section{Electro-Optic Effect in Nitrobenzene}

Nitrobenzene is a straw-yellow-colored liquid that has the highest known Kerr constant. It is very toxic, and care in handling is important. Nitrobenzene has high dielectric strength, high resistivity, and close to $100 \%$ transmission from $420 \mathrm{~nm}$ to the near infrared. ${ }^{6}$ The Kerr constant, dielectric strength, resistivity, and transmission depend on the purity level of nitrobenzene. We used 99\% pure commercially available nitrobenzene with no special purification techniques. The nitrobenzene purity level is not critical if it is used for pulsed measurements; however, for dc measurements special purification techniques are required. ${ }^{1}$

Nitrobenzene is normally isotropic but becomes 
birefringent when placed in an electric field. The medium then behaves optically as if it were an uniaxial medium in which the electric field defines the optic axis. This phenomenon of the electric-fieldinduced birefringence is known as the Kerr electrooptic effect. Because the medium is isotropic, we may choose the applied electric field to be along any coordinate axis. It is customary in electro-optics to choose the $z$ axis as the optic axis, hence we chose the $z$ axis to be in the direction of the applied field. The index ellipsoid for such an arrangement is given by ${ }^{10}$

$x^{2}\left(\frac{1}{n^{2}}+s_{12} E^{2}\right)+y^{2}\left(\frac{1}{n^{2}}+s_{12} E^{2}\right)+z^{2}\left(\frac{1}{n^{2}}+s_{11} E^{2}\right)=1$,

where $E$ is the magnitude of the applied field and $s_{13}=s_{23}=s_{12}$ and $s_{33}=s_{11}$ from the electro-optic tensor. ${ }^{10}$ The index ellipsoid can be written as

$$
\frac{x^{2}+y^{2}}{n_{o}^{2}}+\frac{z^{2}}{n_{e}^{2}}=1
$$

with

$$
\begin{aligned}
& n_{0}=n-1 / 2 n^{3} s_{12} E^{2}, \\
& n_{e}=n-1 / 2 n^{3} s_{11} E^{2} .
\end{aligned}
$$

In general, one would find the birefringence as

$$
n_{e}-n_{o}=1 / 2 n^{3}\left(s_{12}-s_{11}\right) E^{2}
$$

or the more familiar equation,

$$
n_{e}-n_{o}=-K \lambda E^{2}
$$

where

$$
s_{44}=\frac{1}{2}\left(s_{11}-s_{12}\right)=-\frac{K \lambda}{n^{3}},
$$

where $K$ is the Kerr constant, $\lambda$ is the free-space wavelength, and $n$ is the isotropic refractive index of nitrobenzene.

\section{Experimental Arrangement}

Figure 1 shows a block diagram of the experimental arrangement. Plane-polarized light from a $20-\mathrm{mW}$ $\mathrm{He}-\mathrm{Ne}$ laser with the plane of polarization at $45^{\circ}$ in the $x-z$ plane is passed through the beam expansion optics. The expanded beam is split by the input beam splitter B1 located in the analyzer section. Horizontally polarized light gets transmitted and is made to pass through the Kerr cell, where it samples the entire area between the electrodes of the Kerr cell. The light is then directed by mirror M2 onto the output beam splitter B2. The reflected vertically polarized light, which is the reference beam, is passed through a half-wave plate that rotates the polarization to make it horizontal, and mirror M1 directs the

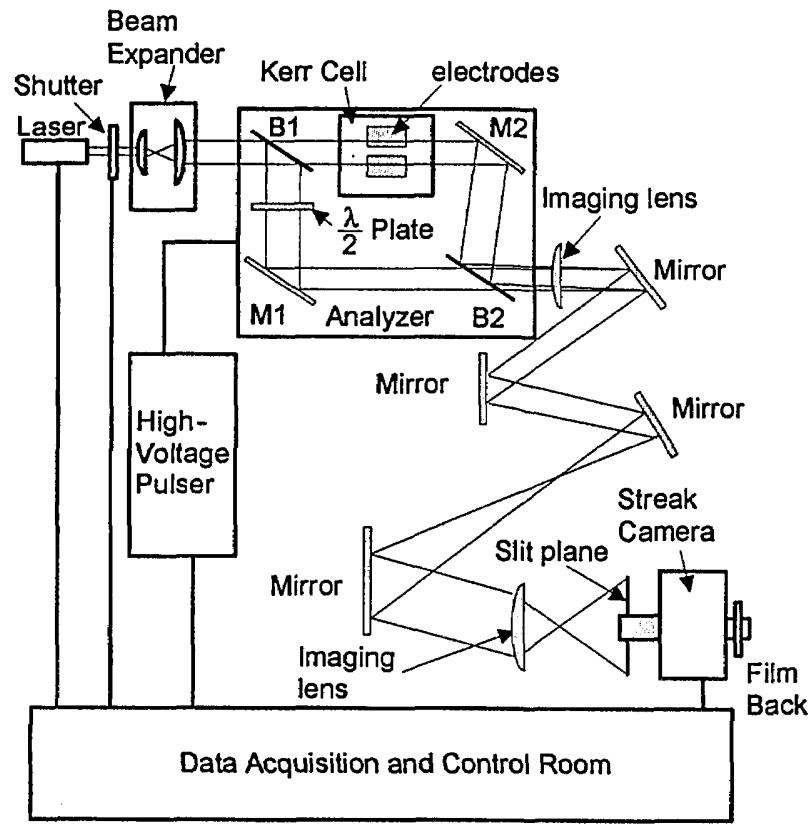

Fig. 1. Schematic diagram of the experimental arrangement.

polarized light onto output beam splitter B2. At the output of beam splitter B2 the two polarization states are made to interfere to produce a sharp fringe image. This fringe image is made incident upon the slit of the streak camera placed in the image plane of the imaging lens system. The slit width corresponds to the electrode gap, and the slit is placed at the center of the fringe image, which corresponds to the center of the electrodes. The sampling beam is shuttered for $5 \mathrm{~ms}$ by an external mechanical shutter to prevent damage to the streak tube and improve the final image on the film. The voltage pulse is applied by a high-voltage pulser that delivers microsecond flattopped voltage pulses across the electrodes. The entire experiment is controlled through the data acquisition and control room, from which the various triggering delays are adjusted and the voltage and current are monitored.

Briefly, the experiment is operated as follows. Initially the various optical pieces are carefully adjusted and the mechanical shutter is then triggered to open, allowing the laser beam to pass through and form the background fringes across the slit of the streak camera. A trigger is then given to the streak camera to initiate the streak recording, and after a certain delay the high-voltage pulser is triggered to apply an 800-ns high-voltage $(0-5-\mathrm{kV})$ pulse across the electrodes. The phase of the sampling beam is changed by the integral of the electric field that is present between the electrodes. ${ }^{11}$ This change in phase causes the fringes to shift in time, and as the streak recording was started before the application of the high-voltage pulse (i.e., when the electric field was zero), the final fringe image on the film is in the form of a continuous flow image of the fringes, with the fringes appearing bent during the time period that the voltage pulse was applied. The output light 
intensity is given by ${ }^{12}$

$$
I \propto \cos ^{2}\left(\frac{\gamma-\Delta_{E}}{2}\right),
$$

where $\gamma$ is the background phase difference (without electric field) and $\Delta_{E}$ is the electric-field-induced phase retardation and is given by ${ }^{12}$

$$
\Delta_{E}=2 \pi \frac{\Delta z}{\delta z}
$$

where $\delta z$ is the background fringe spacing and $\Delta z$ is the induced fringe shift. The fringe shift is measured, and the $s_{11}$ electro-optic coefficient can be calculated by use of the relation ${ }^{12}$

$$
\Delta_{E}=-\frac{\pi L}{\lambda} n^{3} s_{11} E(x, z, t)^{2},
$$

where $L$ is the length of the birefringent path; $\lambda$ is the free-space wavelength; $n$ is the isotropic refractive index, which is 1.53 as given in Ref. 13 for various wavelengths; and $E(x, z, t)$ is the applied electric field.

\section{Experimental Results}

The experiment was conducted with four different applied voltages. Two current-viewing resistors measured the voltage across the electrodes and the current between them, respectively. The voltage and current waveforms were displayed on the oscilloscope and digitized with a CCD camera. Commercial software was then used to determine the values of the digitized waveforms. The current measurement was important for determining if the nitrobenzene medium becomes conductive under high voltage, but no current was observed for any of the applied voltages. The electrodes of the Kerr cell were made of aluminum and were approximately $9 \mathrm{~mm}$ in length and spaced approximately $0.88 \mathrm{~mm}$ apart. The electrode length was measured with a standard vernier caliper. The electrode separation was measured by illuminating the electrode gap and magnifying the image by the use of the imaging lenses. This image was then projected on a plane, and the separation was measured by a metric scale and then divided by the magnifying ratio. Various measurements were taken, and the average was determined. Figure 2 shows the digitized fringe patterns for the different applied voltages. The fringe patterns were first recorded on a high-speed (20,000 ASA) Polaroid film and later digitized with a hand digitizer attached to a computer. The hand digitizer produced a digitized fringe image that could be stored as a file in the computer and later analyzed with a drafting package. In Fig. 2 the vertical axis corresponds to the temporal evolution of the fringes, and the horizontal axis corresponds to the spatial points between the electrodes. As can be observed from Fig. 2(a), with no applied voltage the fringes do not shift. As the applied voltage is increased, the fringes start shifting, with increasing shifts observed for higher voltages.
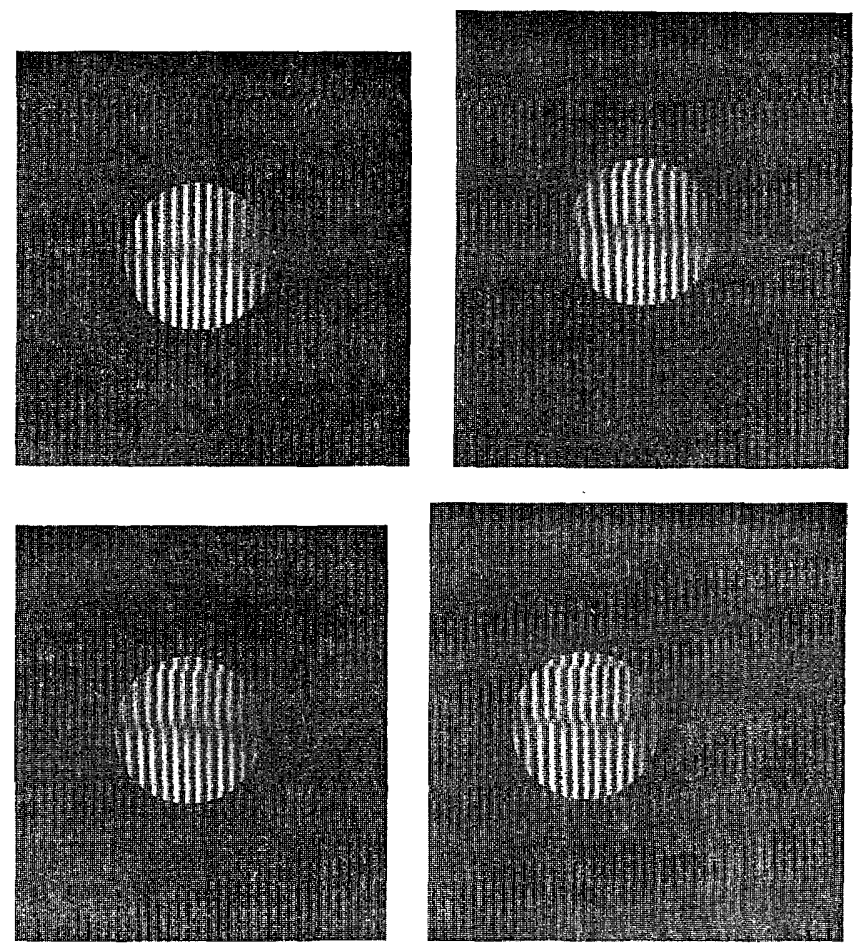

Fig. 2. Fringe patterns for different applied voltages: (a) $0 \mathrm{~V},(\mathrm{~b})$ $2813 \mathrm{~V}$, (c) $3252.5 \mathrm{~V}$, (d) $3616.5 \mathrm{~V}$.

The fringe shift or bending was measured for the central fringe at the center point of the bending. This corresponds physically to the center point between the electrodes and at the center of the applied high-voltage pulse. It can be argued that at that point the electric field is most uniform and can be determined simply by division of the measured voltage by the contact separation. The fringe bending of the central fringe at points close to the center point of the bending was also calculated, and the average was taken. Thus, by determining the fringe bending, one can determine the value of $\Delta_{E}$ in Eq. (9). This value can be then substituted in Eq. (10), and the value of the $s_{11}$ parameter can be determined. By repetition of the procedure for the different applied voltages, the average value can be found. This is shown in Table 1. It should be noted that the value of $s_{11}$ determined here differs from the value reported earlier. ${ }^{14}$ This is attributed to a more accurate fringeshift measurement technique that was not available in the past.

\begin{tabular}{|c|c|c|c|c|c|c|}
\hline $\begin{array}{l}\text { Applied } \\
\text { Voltage }\end{array}$ & $\begin{array}{c}E_{\text {avg }} \\
(\mathrm{V} / \mathrm{cm})\end{array}$ & $\begin{array}{c}\delta z \\
\text { (arb. } \\
\text { units) }\end{array}$ & $\begin{array}{c}\Delta z \\
\text { (arb. } \\
\text { units) }\end{array}$ & $\begin{array}{c}\Delta E \\
\text { (exp.; } \\
\text { (arb. } \\
\text { units) }\end{array}$ & $\begin{array}{c}s_{11} \\
\left(\mathrm{~cm}^{2} / \mathrm{V}^{2}\right) \\
\times 10^{-15}\end{array}$ & $\begin{array}{c}\text { Mean } s_{11} \\
\left(\mathrm{~cm}^{2} / \mathrm{V}^{2}\right) \\
\times 10^{-15}\end{array}$ \\
\hline 3616.52 & 41096.8 & 142.5 & 66.5 & \multicolumn{3}{|c|}{$2.9322-10.848692$} \\
\hline 3252.5 & 36960.2 & 146.167 & 46.33 & 1.9917 & \multicolumn{2}{|c|}{$-9.1099049-9.639826$} \\
\hline 2813 & 31965.9 & 136.667 & 31.833 & 1.4655 & \multicolumn{2}{|l|}{-8.960881} \\
\hline
\end{tabular}

Table 1. Experimental Results to Determine the $s_{11}$ Electro-Optic Coefficient of Nitrobenzene 
The worst-case error in the measured value of the $s_{11}$ coefficient was approximately $25 \%$ and can be attributed to the following factors. The error in the measurement of length that is due to the presence of fringing fields was typically taken as $3 \%$. The error in the measurement of the contact separation was approximately $4.5 \%$. The error in measurement of the fringe shifts was approximately $4 \%$, and this is determined primarily by the ability to distinguish the fringe shifts. The error in the measurement of the voltage was less than $1 \%$, which again depends on the resolution of the CCD camera and the associated software.

We are grateful to Bob Druce and the Lawrence Livermore National Laboratory for loan of the streak camera, Jim Thompson of the University of New Mexico and Z. A. Schelly of the University of Texas at Arlington for useful discussions, and W. E. Dillon of the University of Texas at Arlington for help in analyzing the data. The research was funded by the U.S. Air Force Office of Scientific Research under contract AFOSR 89-0253.

\section{References}

1. H. J. White, "The technique of Kerr cells," Rev. Sci. Instrum. 6, 22-26(1935).

2. H. M. Hertz, "Kerr effect tomography for nonintrusive spatially resolved measurements of asymmetric electric field distributions," Appl. Opt. 25, 914-921 (1986).

3. S. Y. Ettinger and A. C. Venezia, "High voltage pulse measuring system based on Kerr effect," Rev. Sci. Instrum. 34, 221-224 (1963).

4. J. E. Thompson, M. Kristiansen, and M. O. Hagler, "Optical measurement of high electric and magnetic fields," IEEE Trans. Instrum. Meas. IM-25, 1-7 (1976).

5. F. G. Dunnington, "The electrooptical shutter-its theory and techniques," Phys. Rev. 38, 1506-1534 (1931).

6. A. M. Zarem, F. R. Marshall, and F. L. Poole, "An electrooptical shutter for photography," Trans. Am. Inst. Electr. Eng. 68, 84-91 (1949).

7. H. P. Sardesai, W. C. Nunnally, and P. F. Williams, "Surface field measurement of photoconductive power switches using the electro-optic Kerr effect," in Optically Activated Switching, F. J. Zutavern, ed., Proc. Soc. Photo-Opt. Instrum. Eng. 1378, 237-248 (1991).

8. H. P. Sardesai, W. C. Nunnally, and P. F. Williams, "Optical measurements of surface fields on silicon photoconductive power switches," in Proceedings of the Eighth IEEE Pulsed Power Conference, R. White and K. Prestwich, eds. (Institute of Electrical and Electronics Engineers, New York, 1991), pp. 1028-1031.

9. H. P. Sardesai, W. C. Nunnally, and P. F. Williams, "Recent developments in the optical measurement of surface fields on silicon photoconductive power switches," in Emerging Optoelectronic Technologies, A. Selvarajan and K. Shenai, eds., Proc. Soc. Photo-Opt. Instrum. Eng. 1622, 413-417 (1992).

10. A. Yariv and P. Yeh, Optical Waves in Crystals (Wiley, New York, 1984).

11. E. F. Kingsbury, "The Kerr electrostatic effect," Rev. Sci. Instrum. 1, 22-32 (1930).

12. H. P. Sardesai, "Optical measurements of surface electric fields on silicon photoconductors," M.S. thesis (University of Texas at Arlington, Arlington, Tex., 1991).

13. CRC Handbook of Laser Science and Technology, M. J. Weber, ed. (CRC, Boca Raton, Fla., 1986), Vol. 3.

14. H. P. Sardesai, W. C. Nunnally, and P. F. Williams, "An optical technique for measurement of semiconductor surface electric fields," Rev. Sci. Instrum. 63, 3918-3922 (1992). 